\title{
Stability of Solution of the Nonlinear Schrödinger Equation for the Bose-Einstein Condensation
}

\author{
Yeong E. Kim and Alexander L. Zubarev \\ Department of Physics, Purdue University \\ West Lafayette, IN 47907-1397
}

We investigate the stability of the Bose-Einstein condensate (BEC) for the case of atoms with negative scattering lengths at zero temperature using the Ginzburg-Pitaevskii-Gross (GPG) stationary theory. We have found a new exact equation for determining the upper bound of the critical numbers $N_{c r}$ of atoms for a metastable state to exist. Our calculated value of $N_{c r}$ for Bose-Einstein condensation of lithium atoms based on our new equation is in agreement with those observed in a recent experiment.

PACS number(s): 03.75.Fi, 03.65.Db, 05.30.Jp, 67.90.+z

\section{Introduction}

The concept of the Bose-Einstein condensation (BEC) [1] has been known for 73 years, and has been used to describe all physical scales, including liquid ${ }^{4} \mathrm{He}$, exitons in semiconductors, pions and kaons in dense nuclear matter (neutron stars, supernovae), and elementary particles [2]. It is only a few years ago that the BEC phenomenon was observed directly in dilute vapors of alkali atoms, such as rubidium [3], lithium [4,5], and sodium [6], confined in magnetic trap and cooled down to nanokelvin temperatures. At such low temperatures, the thermal de Brogle wavelength increase to submicrometer dimensions. When the scattering length is negative, then nonlinear interaction between atoms is attractive and it has been claimed that the BEC in free space is impossible [7], because the attraction makes the system tend to an ever denser phase. For ${ }^{7} \mathrm{Li}$, the s-wave scattering length $a=(-14.5 \pm$ 0.4) $\AA[8]$. For bosons trapped in an external potential, there may exist a metastable BEC state under certain conditions for a number of atoms below the critical value $N_{c r}$ [9-11]. Stability of the BEC in a trap as a function of nonlinearity has been studied numerically for $\mathrm{T}=0[12-14]$. It was demonstrated $[15,16]$ that global solutions of the Ginzburg-Pitaevskii-Gross equation (GPG) [17] for trapped atoms with negative energies diverge in a finite time. When the system collapses, a more accurate theory is required 
in order to include short range effects.

In this paper, we report our theoretical studies of the stability of the $\mathrm{BEC}$ for the case with negative scattering length at zero temperature using the GPG stationary theory [17]. We have found, for the first time, an exact equation for determination of the upper bound of the $N_{c r}$ for a metastable state to exist.

\section{A necessary condition for a local minimum}

In the mean-field approximation, the ground state energy of the system is given by the GPG energy functional [17]

$$
J(\psi)=\bar{T}+\bar{V}+\frac{g_{o} N}{2} \bar{V}_{H},
$$

with

$$
\begin{gathered}
J(\psi)=E / N, \\
\bar{T}=\int d^{3} r \frac{\hbar^{2}}{2 m}|\nabla \psi|^{2}, \\
\bar{V}=\int d^{3} r \psi^{*} V \psi, \\
\bar{V}_{H}=\int|\psi|^{4} d^{3} r,
\end{gathered}
$$

and

$$
g_{o}=\frac{4 \pi \hbar^{2} a}{m},
$$

where $a$ is the s-wave scattering length, $\mathrm{N}$ is the number of particles in the $\mathrm{BEC}, \mathrm{V}$ is an external trapping potential, and $\psi$ is the condensate ground state wave function normalized as

$$
\int|\psi|^{2} d^{3} r=1
$$

For a harmonic oscillator trap, we have

$$
V(\vec{r})=V_{1}(x)+V_{2}(y)+V_{3}(z),
$$

where 


$$
V_{i}\left(r_{i}\right)=\frac{m}{2} \omega_{i}^{(o) 2} r_{i}^{2}
$$

Using

$$
V_{i}\left(\lambda_{i} r_{i}\right)=\lambda_{i}^{2} V_{i}\left(r_{i}\right),
$$

we can obtain an extremum condition (see Appendix)

$$
\begin{gathered}
2 \bar{T}_{i}-2 \bar{V}_{i}+\frac{g_{o} N}{2} \bar{V}_{H}=0, \\
\bar{T}_{i}=\int d^{3} r \frac{\hbar^{2}}{2 m}\left|\nabla_{i} \psi\right|^{2}, \\
\bar{V}_{i}=\frac{m}{2} \int d^{3} r \psi^{*}(\vec{r})\left(\omega_{i}^{(o) 2} r_{i}^{2}\right) \psi(\vec{r}),
\end{gathered}
$$

and a necessary local minimum condition

$$
\hat{A}>0
$$

where $\hat{A}$ is matrix with matrix elements

$$
A_{i j}=\left(2 \bar{T}_{i}+6 \bar{V}_{i}\right) \delta_{i j}+\left(1-\delta_{i j}\right) \frac{g_{o} N}{2} \bar{V}_{H} .
$$

(See Appendix for details.)

Eqs. (10-12) represent well-known virial theorem equations [12], but the conditions (13) was not discussed in literature, to the best of our knowledge. We note, that if the conditions (13) are not fulfilled, local minimum disappears and solution will be not stable. In the case $a<0$, we can rewrite (14) as

$$
A_{i j}=\frac{\left|g_{o}\right| N \bar{V}_{H}}{2}\left[\tilde{g}_{i} \delta_{i j}+2 \delta_{i j}-1\right]
$$

where

$$
\tilde{g}_{i}=\frac{16 \bar{V}_{i}}{\left|g_{o}\right| N \bar{V}_{H}}
$$

Matrix $\left(2 \delta_{i j}-1\right)$ is not positive (its eigenvaliues are $\left.-1,2,2\right)$ hence in case $V_{1}=V_{2}=V_{3}=0$ (free space) matrix A, is not positive and there is no stable solution.

\section{Upper bounds for $N_{c r}$.}


We consider first an isotropic trap case with

$$
\tilde{g}_{x}=\tilde{g}_{y}=\tilde{g}_{z}=\frac{16}{3} \frac{\bar{V}}{\left|g_{o}\right| N \bar{V}_{H}}
$$

where

$$
\bar{V}=\bar{V}_{x}+\bar{V}_{y}+\bar{V}_{z}=3 \bar{V}_{x}
$$

For this case the local minimum conditions given by Eq. (13) can be written as

$$
\frac{16 \bar{V}}{3\left|g_{o}\right| N \bar{V}_{H}}>1
$$

In terms of $\bar{N}$, which is solution of the following equation

$$
\bar{N}=\frac{16}{3} \frac{\bar{V}}{\left|g_{o}\right| \bar{V}_{n}},
$$

we can state that if $N \geq \bar{N}$, the BEC is not stable. Therefore $\bar{N}$ is an upper bound for $N_{c r}$,

$$
N_{c r} \leq \bar{N}
$$

We note, that Eq.(21) is also valid for anisotropic cases, but the bound given by Eq. (20) in this case can be improved. To show this let us consider an anisotropic harmonics trap

$$
V(\vec{r})=V_{\perp}(\vec{r})+V_{z}(\vec{r}),
$$

here

$$
\begin{gathered}
V_{\perp}(\vec{r})=\frac{m}{2} \omega_{\perp}^{(o) 2}\left(x^{2}+y^{2}\right), \\
V_{z}(\vec{r})=\frac{m}{2} \omega_{z}^{(o) 2} z^{2} .
\end{gathered}
$$

Using Eqs. (22-24) we can rewrite the local minimum conditions (13) as

$$
\frac{4 \bar{V}_{\perp}}{\left|g_{o}\right| N \bar{V}_{H}}+\left(\frac{8}{\left|g_{o}\right| N \bar{V}_{H}}\right)^{2} \bar{V}_{\perp} \bar{V}_{z}-1>0
$$

where

$$
\bar{V}_{\perp}=\frac{m}{2} \omega_{\perp}^{(o) 2} \int \psi^{*}(\vec{r})\left(x^{2}+y^{2}\right) \psi(\vec{r}) d^{3} \vec{r}
$$




$$
\bar{V}_{z}=\frac{m}{2} \omega_{z}^{(o) 2} \int \psi^{*}(\vec{r}) z^{2} \psi(\vec{r}) d^{3} \vec{r} .
$$

Setting the left-hand side of Eq. (25) to zero we obtain the following equation

$$
\bar{N}=\frac{2 \bar{V}_{\perp}}{\left|g_{o}\right| \bar{V}_{H}}\left[1+\left(1+\frac{16 \bar{V}_{z}}{\bar{V}_{\perp}}\right)^{1 / 2}\right]
$$

for upper bound $\bar{N}$

$$
N_{c r} \leq \bar{N}
$$

It should be pointed out that our formulas (28) and (29) are valid for a general anisotropic trap with

$$
\bar{V}_{\perp}=\frac{m}{2} \int \psi^{*}(\vec{r})\left(\omega_{x}^{(o) 2} x^{2}+\omega_{y}^{(o) 2} y^{2}\right) \psi(\vec{r}) d \vec{r} .
$$

To the best of our knowledge, these formulas have never been discussed previously in the literature.

\section{Application to lithium atoms: numerical example}

We assume the trial wave function for $\psi$ in Eqs. (10-28) to be $[14,18]$

$$
\psi_{t}(\vec{r})=\omega_{\perp}^{1 / 2} \omega_{z}^{1 / 2}\left(\frac{m}{\pi \hbar}\right)^{3 / 4} e^{-m\left(\omega_{\perp}^{2}\left(x^{2}+y^{2}\right)+\omega_{z}^{2} z^{2}\right) / 2 \hbar} .
$$

Using Eq. (31), we rewrite Eqs. (10) and (28) as

$$
\begin{gathered}
1-2 \delta_{\perp}^{2}=\delta_{\perp}^{2}\left(1+8 \frac{\delta_{z}}{\delta_{\perp}} \lambda^{2}\right)^{1 / 2} \\
1-\lambda^{2} \delta_{z}^{2}=\delta_{z} \delta_{\perp}\left[1+\left(1+8 \frac{\delta_{z}}{\delta_{\perp}} \lambda^{2}\right)^{1 / 2}\right]
\end{gathered}
$$

and

$$
n=\delta_{z} \delta_{\perp}\left[1+\left(1+8 \frac{\delta_{z}}{\delta_{\perp}} \lambda^{2}\right)^{1 / 2}\right]
$$

where $\delta_{z}=\omega_{\perp}^{(o)} / \omega_{z}, \delta_{\perp}=\omega_{\perp}^{(o)} / \omega_{\perp}, \lambda=\omega_{z}^{(o)} / \omega_{\perp}^{(o)}$, and

$$
n=2\left(\omega_{\perp}^{(o)}\right)^{1 / 2}\left(\frac{m}{2 \pi \hbar}\right)^{1 / 2} \bar{N}|a| .
$$


We find that numerical solution of Eqs. (32-34) for $0 \leq \lambda \leq 1$ can be interpolate as

$$
n=e^{-\left(\alpha+\beta \lambda^{2}\right)}
$$

with $\alpha=0.490419, \beta=0.149175$. Using Eqs. (36) and (35) we have

$$
\bar{N}=\left(\frac{2 \pi \hbar}{\omega_{\perp}^{(o)} m}\right)^{1 / 2} \frac{e^{-\left(\alpha+\beta \lambda^{2}\right)}}{2|a|}
$$

Taking the experimental parameters [5], $\omega_{\perp}^{(o)} / 2 \pi=152 \mathrm{~Hz}$, and $\omega_{z}^{(o)} / 2 \pi=$ $132 \mathrm{~Hz}$, we obtain $\lambda=0.86842$ and $N_{c r} \leq N=1456$. This value of $N_{c r}$ is consistent with theoretical predictions [12-14] and is in agreement with those observed in a recent experiment [5].

\section{Summary and Conclusions}

We have investigated the stability of the BEC for the case of atoms with negative scattering length at zero temperature in a magnetically trapped atomic gas. Using a rigorous derivation, we have found a new exact equation for determining the upper bound of the critical number of atoms $N_{c r}$ for a metastable state to exist. Our calculated value of $N_{c r}$ for BEC of lithium is about 1456 atoms, which is consistent with recent experimental measurements [5] and theoretical predictions [12-14]. 


\section{Appendix}

In this appendix, we give proving Eqs. (10-14). Let us introduce a trial function

$$
\psi_{t}(\vec{r})=\left[\left(1+\epsilon_{1}\right)\left(1+\epsilon_{2}\right)\left(1+\epsilon_{3}\right)\right]^{1 / 2} \psi\left(\left(1+\epsilon_{1}\right) x,\left(1+\epsilon_{2}\right) y,\left(1+\epsilon_{3}\right) z\right),
$$

where $\psi(x, y, z)$ is exact ground state solution of the GPG equation. The evaluation of $J\left(\psi_{t}\right)$, Eq. (1), can be carried out as

$$
\begin{aligned}
J\left(\psi_{t}\right) & \equiv J\left(\epsilon_{1}, \epsilon_{2}, \epsilon_{3}\right)=\left(1+\epsilon_{1}\right)^{2} \bar{T}_{x}+\left(1+\epsilon_{2}\right)^{2} \bar{T}_{y}+\left(1+\epsilon_{3}\right)^{2} \bar{T}_{z}+ \\
+\frac{1}{\left(1+\epsilon_{1}\right)^{2}} \bar{V}_{x} & +\frac{1}{\left(1+\epsilon_{2}\right)^{2}} \bar{V}_{y}+\frac{1}{\left(1+\epsilon_{3}\right)^{2}} \bar{V}_{z}+\left(1+\epsilon_{1}\right)\left(1+\epsilon_{2}\right)\left(1+\epsilon_{3}\right) \frac{g_{o} N}{2} \bar{V}_{H}
\end{aligned}
$$

where

$$
\bar{T}_{i}=\int d^{3} r \frac{\hbar^{2}}{2 m}\left|\nabla_{i} \psi\right|^{2}
$$

and

$$
\bar{V}_{i}=\frac{m}{2} \int d^{3} r \psi^{*}(\vec{r})\left(\omega_{i}^{(o) 2} r_{i}^{2}\right) \psi(\vec{r})
$$

Since $\psi(\vec{r})$ is exact ground state solutions, $J\left(\epsilon_{1}, \epsilon_{2}, \epsilon_{3}\right)$ has an extremum at $\epsilon_{1}=\epsilon_{2}=\epsilon_{3}=0$, which requires that

$$
\left.\frac{\partial J\left(\epsilon_{1}, \epsilon_{2}, \epsilon_{3}\right)}{\partial \epsilon_{i}}\right|_{\epsilon_{1}=\epsilon_{2}=\epsilon_{3}=0}=0
$$

Substitution (A.2) into (A.3) yields Eq. (10),

$$
2 \bar{T}_{i}-2 \bar{V}_{i}+\frac{g_{o} N}{2} \bar{V}_{H}=0 .
$$

Eq. (A.4) represents well-known virial theorem equations [12].

Now, let us introduce matrix $\hat{A}$ with matrix elements

$$
A_{i j}=\left.\frac{\partial^{2} J\left(\epsilon_{1}, \epsilon_{2}, \epsilon_{3}\right)}{\partial \epsilon_{i} \partial \epsilon_{j}}\right|_{\epsilon_{1}, \epsilon_{2}, \epsilon_{3}=0}=\left(2 \bar{T}_{i}+6 \bar{V}_{i}\right) \delta_{i j}+\left(1-\delta_{i j}\right) \frac{g_{o} N}{2} \bar{V}_{H} .
$$

In terms of $\hat{A}$ we can write a local minimum conditions as

$$
\hat{A}>0
$$




\section{References}

[1] S. N. Bose, Z. Phys. 26, 178 (1924); A. Einstein, Sitz. Press Akad. Wiss. 1924, 3 (1924).

[2] A. Griffin, D. Snoke, and S. Stringari, Bose-Einstein Condensation (Cambridge, New York, 1995).

[3] M. H. Anderson, J. R. Ensher, M. R. Matthews, C. E. Wieman, and E. A. Cornell, Science 269, 198 (1995).

[4] C. C. Bradley, C. A. Sackett, J. J. Tollett, and R. G. Hulet, Phys. Rev. Lett.. 75, 1687 (1995).

[5] C. C. Bradley, C. A. Sackett, and R. G. Hulet, Phys. Rev. Lett. 78, 985 (1997).

[6] K. B. Davis, M.-O. Mewes, M. R. Andrew, N. J. Van Druten, D. S. Durfes, D. M. Kurn, and W. Ketterle, Phys. Rev. Lett. 75, 3969 (1995).

[7] T. D. Lee, K. Huang, and C. N. Yang, Phys. Rev. 106, 1135 (1957).

[8] E. R. I. Abraham, W. I. McAlexander, C. A. Sackett, and R. G. Hulet, Phys. Rev. Lett. 74, 1315 (1995).

[9] Y. Kagan, G. V. Shlyapnikov, and J. T. M. Walraven, Phys. Rev. Lett. 76, 2670 (1996).

[10] H. T. C. Stoof, J. Stat. Phys. 87, 1353 (1997).

[11] E. V. Shuryak, Phys. Rev. A 54, 3151 (1996).

[12] F. Dalfovo and Stringari, Phys. Rev. A53, 2477 (1996).

[13] R. J. Dodd, M. Edwards, C. J. Williams, C. W. Clark, M. J. Holland, P. A. Ruprecht, and K. Burnett, Phys. Rev. A54, 661 (1996).

[14] Hualin Shi and Wei-Mou Zheng, Phys. Rev. A55, 2930 (1997).

[15] L. P. Pitaevskii, Report cond.-mat./9605119.

[16] Kimitaka Watanabe, Tetsuga Mukai, and Takaaki Mukai, Phys. Rev. A55, 3639 (1997).

[17] L. Ginzburg, and L. P. Pitaevskii, Zh. Eksp. Teor. Fiz. 34, 1240 (1958)

[Sov. Phys. JETP 7, 858 (1958)]; E. P. Gross, J. Math. Phys. 4, 195 (1963).

[18] G. Baym and C. J. Pethick, Phys. Rev. Lett. 76, 6 (1996). 